\title{
Acetylcholine revisited
}

\section{Anders Björklund and Stephen B. Dunnett}

IF impaired cortical cholinergic function associated with damage to the basal forebrain cholinergic neuron system is instrumental in dementia-related cognitive decline $^{1,2}$, then restoration of forebrain cholinergic neurotransmission might be enough to improve at least some aspects of impaired learning and memory, particularly in conditions such as Alzheimer's disease. The neurotransmitter acetylcholine $(\mathrm{ACh})$ is suspected to be an important participant in the maintenance of normal cognitive function, but it is not clear to what extent deficits seen after basal forebrain lesions can be attributed to loss of cortical cholinergic neurotransmission.

\section{Recovery}

Now, on page 484 of this issue, Winkler et al. ${ }^{3}$ show that, in rats, supplying $\mathrm{ACh}$ to the fronto-parietal cortex, achieved by transplantation of fibroblasts engineered to release $\mathrm{ACh}$ in a constitutive manner, can alleviate behavioural deficits induced by excitotoxic lesions of the forebrain cholinergic neurons located in the nucleus basalis magnocellularis (NBM). On the basis of their results, the authors propose that the presence of $\mathrm{ACh}$ in the neocortex could be essential for the recovery of spatial memory.

This study is of particular interest in that it is the first to use cells engineered by $e x$ vivo gene transfer techniques to explore the role of cholinergic mechanisms in functional recovery after damage to the central nervous system. The results are important for two reasons. First, functional recovery was obtained by implants of cells that secrete $\mathrm{ACh}$ locally in a non-regulated, constitutive manner, whereas the NBM lesions are known to disrupt long-distance connections from subcortical areas and remove synaptic regulation of intrinsic cortical circuits. Second, recovery was seen after focal placements of the ACh cell transplants into the fronto-parietal cortex (six on each side), whereas the non-ACh-producing control cells had no effect. This observation, which is consistent with results from intracortical transplants of cholinergic neuroblasts from the fetal basal forebrain ${ }^{4-6}$, suggests that local $\mathrm{ACh}$ delivery to restricted areas of the neocortex may be sufficient to induce significant functional recovery in this model. These findings are pertinent to our understanding of the mechanisms of subcortical cholinergic regulation of cortical function: in particular, they suggest that cortical circuits may depend upon diffuse cholinergic activation to sustain their basic function, rather than upon temporally or spatially patterned inputs relayed via afferent neuronal connections.

This brings us to what the new study ${ }^{3}$ can tell us about the normal role of the forebrain cholinergic system in cognitive function. Interpretation of the data of Winkler et al. is by no means straightforward in this respect. There is the question of specificity at three levels - whether the functional deficit induced by excitotoxic NBM lesions (and, by inference, in Alzheimer's dementia) is indeed cholinergic, cortical and cognitive in nature, which may not be the case. In fact, neither the lesion nor the behavioural task used in this study indicates a degree of specificity that would allow any firm conclusions.

Detailed analysis of the damage induced by injections of excitotoxic amino acids into the NBM has shown that both cholinergic and non-cholinergic neurons are affected and that ibotenic acid, the toxin used here, damages pallidal neurons as much as cholinergic neurons ${ }^{7}$. Indeed, more selective injury to NBM cholinergic neurons by quisqualic acid, AMPA or the saporin-192-IgG immunotoxin is associated with minimal deficits in the watermaze task, even though cholinergic denervation of the neocortex is more extensive than that caused by ibotenic acid ${ }^{8,9}$.

The available evidence thus favours the view that the deficits in water-maze learning seen after NBM lesions in the rat are as likely to be attributable to damage in efferent pathways of the neostriatum as of cholinergic projections to the neocortex, and are as likely to involve some form of sensorimotor dysfunction or attentional disturbance as any impairment in cognitive function ${ }^{10}$. This issue needs a wider repertoire of behavioural tasks, as well as more specific cholinergic lesions, before it can be resolved.

We can conclude, therefore, that the behavioural deficits in NBM-lesioned rats may not be purely cholinergic, nor entirely cortical, nor cognitive in nature. But the available data indicate that local supply of $\mathrm{ACh}$ to the denervated cortex is sufficient to promote significant functional recovery in this model. Previous studies using transplants of developing forebrain cholinergic neurons from rat embryos were based on the idea that fetal cholinergic neurons may be able to substitute both structurally and functionally for the lost cholinergic afferents in NBM-lesioned animals. The new results suggest that impulse-dependent, regulated synaptic release may not be necessary for $\mathrm{ACh}$ to induce functional recovery.

In fact, Winkler et al.'s genetically engineered fibroblasts release $\mathrm{ACh}$ (and choline) in a non-regulated, constitutive manner. The fact that only the fibroblasts bearing the choline acetyltransferase gene were effective does suggest that the observed effect was due to ACh release and activation of cholinergic receptors in the area surrounding the transplants. This is compatible with studies in which deficits associated with forebrain cholinergic damage are alleviated by cholinomimetic drugs such as physostigmine or tacrine. Moreover, non-cholinergic drugs that act to enhance cortical function in a more general way can provide a similar recovery of the behavioural deficits associated with NBM lesions and other types of cholinergic blockade ${ }^{11,12}$. This suggests that a pharmacological reversal of deficits acting at the neocortical level can be mediated by as-yet rather ill-defined processes of 'cognitive enhancement', and does not necessarily indicate that the underlying deficit is itself cholinergic. An alternative explanation for the present results could be that, rather than acting as a replacement for the lost cholinergic innervation, $\mathrm{ACh}$ secreted by the engineered cell grafts acts in the host neocortex to promote the animal's ability to compensate for the lesion-induced injury.

\section{Message}

Although the mechanisms underlying behavioural recovery after brain damage are complex, this should not distract from the central message of Winkler et al., namely that genetically engineered cells can be used to provide a local supply of biologically active molecules to an affected brain region in order to promote functional recovery and repair. This approach should provide new experimental tools for cell transplantation in animal disease models and offers promise for the development of prospective restorative therapies of brain damage and neurodegenerative disease.

Anders Björklund is in the Department of Medical Cell Research, University of Lund, S-223 62 Lund, Sweden. Stephen B. Dunnett is in the MRC Cambridge Centre for Brain Repair, and the Department of Experimental Psychology, University of Cambridge, Cambridge CB2 2PY, UK.

1. Drachman, D. A. \& Sahakian, B. J. in The Psychobiology of Aging: Problems and Perspectives (ed. Stein, D.) 347-368 (Elsevier, Amsterdam, 1980).

2. Coyle, J. T., Price, D. L. \& DeLong, M. R. Science 219, $1184-1190(1983)$

3. Winkler, J., Suhr, S. T., Gage, F. H., Thal, L. J. \& Fisher, L. J. Nature 375, 484-487 (1995).

4. Fine, A., Dunnett, S. B., Björklund, A. \& Iversen, S. D Proc. natn. Acad. Sci.U.S.A. 82, 5227-5230 (1985)

5. Dunnett, S. B. et al. Neuroscience 16, 787-797 (1985)

6. Hodges, H. et al. Neuroscience 45, 587-607 (1991)

7. Page, K. J et al. Neuroscience 43, 457-472 (1991).

8. Dunnett, S. B., Everitt, B. J. \& Robbins, T. W. Trends Neurosci. 14, 494-501 (1991).

9. Gallagher, M. \& Colombo, P. J. Curr. Opin. Neurobiol. 5. 161-168(1995).

10. Whishaw, I. Q., Mittleman, G., Bunch, S. T. \& Dunnett, S. B. Behav. Brain Res. 24, 125-138 (1987).

11. Sarter, M. Bruno, J. P. \& Dudchenko, P. Psychopharmacology 101, 1-17 (1990).

12. Mondadori, C. Behav. Brain Res. 59, 1-9 (1993). 